\title{
Características fenológicas e físicas e perfil de ácidos graxos em oliveiras no sul de Minas Gerais
}

\author{
Marcelo Caetano de Oliveira(1), José Darlan Ramos( ${ }^{(1)}$, Rafael Pio(1) e Maria das Graças Cardoso(2) \\ (1)Universidade Federal de Lavras (Ufla), Departamento de Agricultura, Caixa Postal 3.037, CEP $37200-000$ Lavras, MG. \\ E-mail: caetanocaldas@hotmail.com, darlan@dag.ufla.br, rafaelpio@hotmail.com ${ }^{(2)}$ Ufla, Departamento de Química. E-mail: mcardoso@dqi.ufla.br
}

Resumo - O objetivo deste trabalho foi avaliar as características fenológicas e físicas, o teor lipídico e o perfil de ácidos graxos dos frutos de cultivares de oliveira, da Serra da Mantiqueira, sul do Estado de Minas Gerais. Foram avaliadas as cultivares Alto D’Ouro, Arbequina, Galega, Grappolo 541, Grappolo 561, Grappolo 575, Negroa, Penafiel, Ropades 392 e Santa Catalina, nos anos agrícolas 2008/2009, 2009/2010 e 2010/2011. Extraiu-se o óleo bruto das cultivares ricas em lipídeos, para a avaliação do perfil de ácidos graxos. O florescimento ocorre de julho a outubro, e a colheita de janeiro a março. A cultivar mais precoce é a Arbequina, e as mais tardias são Galega, Grappolo 575 e Santa Catalina. 'Grappolo 561' apresenta frutos de maior diâmetro transversal, e 'Grappolo 541' frutos de maior massa total. 'Arbequina' e 'Grappolo 561' apresentam maior teor de lipídeos.

Termos para indexação: Olea europaea, azeite de oliva, ciclo reprodutivo.

\section{Phenological and physical characteristics and fatty acids of olive trees from the south of Minas Gerais, Brazil}

\begin{abstract}
The objective of this work was to evaluate the phenological and physical characteristics, the lipid content and the fatty acid profile of fruit of olive trees from Serra da Mantiqueira, at Minas Gerais state, Brazil. The cultivars Alto D'ouro, Arbequina, Galega, Grappolo 541, Grappolo 561, Grappolo 575, Negroa, Penafiel, Ropades 392 and Santa Catalina were evaluated in the 2008/09, 2009/10 and 2010/11 cycles. For fatty acids profile evaluation, crude oil was extracted from lipid-rich cultivars. The flowering ranges from July to October, and the harvest from January to March. The earliest cultivar is 'Arbequina', and the latest ones are 'Galega', 'Grappolo 575' and 'Santa Catalina'. 'Grappolo 561' has fruit with the longest transverse diameter, and 'Grappolo 541' has fruit with the highest total mass. 'Arbequina' and 'Grappolo 561' show the highest lipid content.
\end{abstract}

Index terms: Olea europaea, olive oil, reprodutive cycle.

\section{Introdução}

O Brasil é considerado um dos maiores importadores mundiais de azeitonas e derivados. Segundo dados da Companhia Nacional de Abastecimento (Indicadores da agropecuária, 2010), em 2009, foram importados aproximadamente 44 mil toneladas de azeite e 70 mil toneladas de azeitona em conservas, o que movimenta mais de um bilhão de reais no mercado nacional com esses produtos. Isto decorre principalmente do fato de o azeite preservar suas características químicas, que beneficiam a saúde, depois de extraído do fruto (Oliveira et al., 2010a).

As inúmeras cultivares de oliveira, plantadas atualmente, são oriundas de cruzamentos e seleções de materiais cultivados desde períodos remotos; assim, esta diversidade necessita de ser avaliada em condições específicas de ambiente, principalmente nas áreas mais altas da Serra da Mantiqueira, local em que se iniciou o recente cultivo de oliveiras no Brasil e que tem aumentado o interesse dos produtores (Vieira Neto et al., 2011).

Para uma produção satisfatória da oliveira, são necessárias, entre outros fatores, temperaturas amenas durante o inverno e amplitude térmica, em que a planta passa por um período de dormência para a indução floral (Cordeiro et al., 2001), pois a oliveira é oriunda de regiões com clima mediterrâneo, com inverno rigoroso e verão quente e seco; seu crescimento é paralisado, quando a temperatura fica abaixo de $12,5^{\circ} \mathrm{C}$, momento em que ocorre o acúmulo de frio necessário à reprodução. Quanto à frutificação, a temperatura 
adequada varia entre $25^{\circ} \mathrm{C}$ e $35^{\circ} \mathrm{C}$, no entanto, as oliveiras são capazes de suportar temperaturas próximas a $40^{\circ} \mathrm{C}$, sem que os ramos e folhas sofram queimaduras (Coutinho et al., 2009).

Oliveira et al., 2010b avaliaram o comportamento de uma coleção de genótipos, adaptados às condições sul-mineiras, e obtiveram até o momento resultados promissores, com indivíduos que se destacam no florescimento e produção regular de frutos.

Um ponto importante é o rendimento em óleo, em razão do pressuposto de que cultivares produtoras de frutos com maiores dimensões promovem rendimento superior de azeite, o que resultaria em aumento na produtividade de óleo por área cultivada. Outro ponto relevante, quanto ao aumento da produção de frutos destinados à extração de azeite, é o emprego de cultivares polinizadoras, que podem propiciar aumento significativo na produtividade das oliveiras (Suárez \& Rallo, 1987). Neste caso, há necessidade de se conhecer a descrição fenológica das cultivares de oliveira nas condições sul-mineiras, para definição e plantios que garantam a sincronia floral.

Para se verificar a qualidade do azeite obtido, o mais indicado é a análise da composição em ácidos graxos, por meio de cromatografia gasosa, que permite diagnosticar divergências não especificadas na embalagem e não detectáveis pelos métodos convencionais, como coloração, acidez e índice de peróxidos (Tsimidou, 1998).

O objetivo deste trabalho foi avaliar as características fenológicas e fisicas, o teor lipídico e o perfil de ácidos graxos dos frutos de diferentes cultivares de oliveira, nas condições das áreas altas da Serra da Mantiqueira, no sul de Minas Gerais.

\section{Material e Métodos}

O experimento foi realizado de janeiro de 2008 a março de 2011, na Fazenda Experimental de Maria da Fé, da Empresa de Pesquisa Agropecuária de Minas Gerais, em Maria da Fé - a $22^{\circ} 18^{\prime} \mathrm{S}, 4^{\circ} 23^{\prime} \mathrm{W}$, e altitude média de $1.276 \mathrm{~m}$, microrregião da Serra da Mantiqueira, ao sul do Estado de Minas Gerais. O solo foi classificado como Latossolo Vermelho distrófico conforme Santos et al. (2006). O clima é mesotérmico, com temperatura média de $17^{\circ} \mathrm{C}$ e precipitação $1.738,6 \mathrm{~mm}$ anuais. Entre os anos de 2008 e 2010, a quantidade de unidades de frio, abaixo de $7,2^{\circ} \mathrm{C}$, durante o inverno, foi cerca de $600^{\circ} \mathrm{C}$, suficiente para as oliveiras florescerem e produzirem (Vieira Neto et al., 2008).

As cultivares de oliveira avaliadas neste trabalho foram: Alto D'Ouro, Arbequina, Galega, Grappolo 541, Grappolo 561, Grappolo 575, Negroa, Penafiel, Ropades 392 e Santa Catalina, todas com 18 anos de idade, mantidas em espaçamento $7 \times 7 \mathrm{~m}$, sem o emprego de irrigação. Utilizou-se o delineamento de blocos ao acaso, com dez tratamentos (cultivares), quatro blocos e três plantas por parcela. Durante a condução do experimento, as plantas foram adubadas, de acordo com a análise do solo e recomendações de Viera Neto et al. (2008), e foram pulverizadas sistematicamente com defensivos agrícolas específicos e podadas para limpeza e arejamento.

Nos anos agrícolas de 2008/2009, 2009/2010 e 2010/2011, registraram-se as seguintes características fenológicas: início da floração (emissão do botão floral); abertura das flores ( $5 \%$ de flores abertas); plena floração ( $70 \%$ de flores abertas); final de floração (95\% ou mais de flores abertas); início da frutificação ( $5 \%$ de frutos pegos); e início e final de colheita. Para isso, foram realizadas observações diárias na copa das plantas pertencentes ao experimento, durante o período reprodutivo, nos três ciclos avaliados.

Para as análises físicas e químicas, as azeitonas foram colhidas em fevereiro de 2009, quando se apresentavam no ponto indicado para extração do azeite (coloração passando de verde para preto). Os comprimentos longitudinais e transversais foram mensurados em 100 frutos frescos, em quatro repetições de 25 frutos de cada cultivar. Posteriormente, obteve-se a massa média dos frutos frescos, da polpa e da semente. $\mathrm{O}$ teor de lipídeos foi determinado na massa triturada (polpa e caroço), com uso do extrator de Soxhlet TE 044 (Tecnal Equipamentos para Laboratório, Piracicaba, SP, Brasil), pelo método gravimétrico, baseado na perda de peso do material submetido à extração com éter etílico ou na quantidade de material solubilizado pelo solvente, conforme Horwitz (2005).

Para as análises físico-químicas dos frutos, utilizou-se o delineamento experimental inteiramente casualizado, com dez tratamentos (cultivares) e quatro repetições.

Para a determinação do perfil de ácidos graxos, $10 \mathrm{~kg}$ de frutos, das cultivares Arbequina e Grappolo 561, que apresentaram o maior teor de lipídeos neste trabalho 
(além do azeite espanhol extravirgem), passaram por prensagem hidráulica, em que o óleo em estado bruto foi extraído por compressão das azeitonas colhidas há menos de 24 horas. Como testemunha, foi utilizada uma amostra de azeite espanhol extravirgem, presente no comércio local. Em seguida, amostras de óleo bruto de $5 \mathrm{~mL}$, das cultivares e testemunha, foram esterificadas conforme Hartman \& Lago (1973). A determinação da composição de ácidos graxos foi determinada por cromatografia de fase gasosa, com cromatógrafo Shimadzu GC-17A V3 (Shimadzu Corp., Kyoto, Japão), equipado com detector de ionização de chama e coluna capilar de polietilenoglicol DB-Wax com $30 \mathrm{~m}$ de comprimento, $0,25 \mathrm{~mm}$ de diâmetro interno, 0,25 micrometro de espessura.

As condições cromatográficas utilizadas foram: temperatura inicial da coluna igual a $60^{\circ} \mathrm{C}$ por $1 \mathrm{~min}$, aumentada à taxa de $30^{\circ} \mathrm{C}$ por min, até $180^{\circ} \mathrm{C}$ por $5 \mathrm{~min}$, com aquecimento de $3^{\circ} \mathrm{C}$ por min até temperatura final da coluna de $230^{\circ} \mathrm{C}$ durante $14 \mathrm{~min}$, modo split à razão de 1:20. O gás de arraste usado foi o nitrogênio, em fluxo de $2,74 \mathrm{~mL}$ por min. A temperatura do injetor foi de $230^{\circ} \mathrm{C}$, e a do detector de $250^{\circ} \mathrm{C}$. A identificação dos ácidos graxos foi realizada por comparação entre os tempos de retenção das amostras e dos padrões.

Utilizou-se o delineamento experimental inteiramente casualizado, com três tratamentos: azeites extraídos das cultivares Arbequina e Grappolo 561, azeite espanhol extravirgem comercial e três repetições.

Os resultados obtidos foram submetidos à análise de variância, a 5\% de probabilidade, pelo teste de Tukey, para comparações múltiplas.

\section{Resultados e Discussão}

No ano agrícola 2008/2009, o início da floração se deu a partir da primeira quinzena do mês de agosto, e se estendeu até a primeira quinzena de setembro, em que 'Arbequina' e 'Grappolo 575' mostraram-se precoces, e 'Galega', tardia (Tabela 1). Em 2009/2010, notou-se antecipação no início do florescimento na maioria das cultivares, da segunda quinzena de julho até a primeira quinzena de agosto. No terceiro ciclo de avaliação (2010/2011), o início da floração ocorreu entre a segunda quinzena de julho, nas oliveiras 'Arbequina' e 'Grappolo 541', e na primeira quinzena de setembro na 'Grappolo 561', 'Grappolo 575' e 'Santa Catalina'. Nos três ciclos avaliados, observou-se que o início da floração concentrou-se nos meses de julho e agosto, na maioria das cultivares, com diferença no ciclo de até 49 dias.

A abertura de flores ocorreu entre a primeira quinzena de agosto e a segunda quinzena de setembro, enquanto a plena floração ficou concentrada no mês de setembro, na maioria das cultivares, nos três ciclos avaliados. Apesar de algumas cultivares, como a Arbequina, serem consideradas autógamas, a diversificação de genótipos na mesma área de cultivo pode favorecer a frutificação e, consequentemente, elevar a produtividade (Suárez \& Rallo, 1987). Nesse sentido, é interessante a inclusão

Tabela 1. Período de floração de cultivares de oliveira, nos anos agrícolas 2008/2009, 2009/2010 e 2010/2011 em Maria da Fé, MG.

\begin{tabular}{|c|c|c|c|c|c|}
\hline Cultivar & IF & $\mathrm{AF}$ & $\mathrm{PF}$ & $\mathrm{FF}$ & IF-FF (dias) \\
\hline & \multicolumn{5}{|c|}{ Ano agrícola 2008/2009 } \\
\hline Alto D'Ouro & 11/ago. & 01/set. & $30 /$ set. & 10/out. & 60 \\
\hline Arbequina & 05/ago. & 20/ago. & $10 /$ set. & $30 /$ set. & 56 \\
\hline Galega & 01/set. & $25 /$ set. & 05/out. & 20/out. & 49 \\
\hline Grappolo 541 & 11/ago. & 21/ago. & $17 /$ set. & 04/out. & 54 \\
\hline Grappolo 561 & 11/ago. & 20/ago. & $30 /$ set. & 30/out. & 80 \\
\hline Grappolo 575 & 02/ago. & 21/ago. & 07/set. & $24 /$ set. & 53 \\
\hline Negroa & 11/ago. & 20/ago. & $15 /$ set. & 10/out. & 60 \\
\hline Penafiel & 11/ago. & 21/ago. & $13 /$ set. & $30 /$ set. & 50 \\
\hline Ropades 392 & 10/ago. & 20/ago. & $15 /$ set. & 05/out. & 56 \\
\hline \multirow[t]{2}{*}{ Santa Catalina } & 20/ago. & $10 /$ set. & $30 /$ set. & 29/out. & 70 \\
\hline & \multicolumn{5}{|c|}{ Ano agrícola 2009/2010 } \\
\hline Alto D'Ouro & 05/ago. & 25/ago. & $10 /$ set. & 10/out. & 66 \\
\hline Arbequina & 05/ago. & 20/ago. & 10/set. & 30/set. & 56 \\
\hline Galega & 25/jul. & 20/ago. & 05/set. & $20 /$ set. & 57 \\
\hline Grappolo 541 & 15/jul. & 16/ago. & $13 /$ set. & 05/out. & 82 \\
\hline Grappolo 561 & 15/jul. & 25/ago. & $20 /$ set. & 08/out. & 85 \\
\hline Grappolo 575 & 15/jul. & 10/ago. & $10 /$ set. & 08/out. & 85 \\
\hline Negroa & 10/ago. & 30/ago. & $15 /$ set. & 10/out. & 61 \\
\hline Penafiel & 20/jul. & 10/ago. & 30/ago. & $30 /$ set. & 72 \\
\hline Ropades 392 & 26/jul. & 18/ago. & $10 /$ set. & 30/set. & 66 \\
\hline \multirow[t]{2}{*}{ Santa Catalina } & 07/ago. & 30/ago. & $25 /$ set. & 15/out. & 69 \\
\hline & \multicolumn{5}{|c|}{ Ano agrícola 2010/2011 } \\
\hline Alto D'Ouro & 20/ago. & 10/set. & 23/set. & 10/out. & 51 \\
\hline Arbequina & 27/jul. & 20/ago. & 08/set. & 30/set. & 65 \\
\hline Galega & 20/ago. & $10 /$ set. & $25 /$ set. & 10/out. & 51 \\
\hline Grappolo 541 & 20/jul. & 10/set. & $25 /$ set. & 12/out. & 84 \\
\hline Grappolo 561 & $05 /$ set. & 18/set. & $30 /$ set. & 20/out. & 45 \\
\hline Grappolo 575 & $05 /$ set. & $15 /$ set. & $30 /$ set. & 10/out. & 35 \\
\hline Negroa & 20/ago. & 08/set. & $22 /$ set. & 10/out. & 51 \\
\hline Penafiel & 30/ago. & $10 /$ set. & $22 /$ set. & 05/out. & 36 \\
\hline Ropades 392 & 30/ago. & $15 /$ set. & $25 /$ set. & 08/out. & 39 \\
\hline Santa Catalina & $05 /$ set. & $20 /$ set. & 29/set. & 15/out. & 40 \\
\hline
\end{tabular}

IF, início da floração; AF, abertura de flores ( $5 \%$ de flores abertas); PF, plena floração (mais de 70\% flores abertas); FF, final de floração (mais de 95\% de flores abertas); IF-FF, período de floração. 
de mais cultivares de produção de azeitonas, para a extração de azeite, nas áreas de cultivo no sul de Minas. Baixa frutificação efetiva ou baixa produção, muitas vezes, pode ser decorrente de polinização deficiente, em razão da escolha inadequada dos polinizadores e não coincidência de floração entre as cultivares utilizadas. Assim, o plantio da cultivar Arbequina, associada à Grappolo 541 ou Grappolo 575, por exemplo, pode favorecer expressivamente a elevação da produtividade por área.

O término do período de floração, no ano agrícola (2008/2009), ocorreu entre a segunda quinzena de setembro e o final de outubro, e as cultivares Grappolo 575, Arbequina e Penafiel mostraram-se mais precoces, e Grappolo 561 e Santa Catalina, tardias. No ano agrícola 2009/2010, o final da floração ocorreu entre a segunda quinzena de setembro e a primeira de outubro, tendo sido 'Galega', 'Arbequina', 'Penafiel' e 'Ropades 392' as mais precoces, e 'Santa Catalina' e 'Alto D'Ouro' tardias. No ano agrícola 2010/2011, o final da floração se concentrou no mês de outubro, na 'Penafiel' e na 'Arbequina' que foram as mais precoces, e na 'Grappolo 561' e 'Santa Catalina' que foram as mais tardias (Tabela 1). Na média dos três ciclos, o período de floração foi de 54 dias, em que a plena floração se concentrou nos meses de setembro e outubro, em todas as cultivares.

O período de florescimento é fortemente influenciado pelas condições ambientais. Em condições de inverno ameno, as cultivares oriundas de regiões de clima temperado, como a oliveira, podem apresentar grande variabilidade quanto ao período de floração de um ciclo para outro (Petri et al., 2008), em razão da instabilidade térmica durante o período invernal, característica de regiões subtropicais (Bettiol Neto et al., 2011) como o sul de Minas. No Brasil, ainda há poucos estudos sobre o comportamento fenológico das cultivares.

Verificou-se que o início da frutificação ficou concentrado entre a segunda quinzena de agosto e a primeira de outubro, e que 'Arbequina' e 'Galega 575' se mostraram mais precoces, nos três anos agrícolas avaliados. O início da frutificação ocorreu em setembro na maioria das cultivares avaliadas (Tabela 2).

O período de desenvolvimento dos frutos variou entre 144 e 192 dias, em que 'Grappolo 575' apresentou o maior ciclo de desenvolvimento e Alto D'ouro o menor (Tabela 2).
Em relação ao período de colheita, observouse concentração entre o final de janeiro e início de março, e o mês de fevereiro foi o período de maior concentração dos três ciclos, com duração média de 24 dias (Tabela 2). O menor período de colheita ocorreu no primeiro e terceiro ciclos de avaliação, com até 11 dias para 'Grappolo 561' e 'Alto D'ouro'; os maiores períodos foram obtidos no primeiro e segundo ciclos, com 26 e 31 dias, na cultivar Arbequina.

Tabela 2. Período de frutificação em cultivares de oliveira, nos anos agrícolas 2008/2009, 2009/2010 e 2010/2011 em Maria da Fé, MG.

\begin{tabular}{|c|c|c|c|c|c|}
\hline \multirow[t]{2}{*}{ Cultivar } & IFR & IC & $\mathrm{FC}$ & IC-FC & IFR-FC \\
\hline & & & & & lias)------ \\
\hline & \multicolumn{5}{|c|}{ Ano agrícola 2008/2009 } \\
\hline Alto D’Ouro & $15 /$ set. & $10 /$ fev. & $27 /$ fev. & 17 & 165 \\
\hline Arbequina & 30/ago. & $30 /$ jan. & $25 /$ fev. & 26 & 179 \\
\hline Galega & 05/out. & $28 /$ fev. & $17 /$ mar. & 17 & 163 \\
\hline Grappolo 541 & $15 /$ set. & $10 /$ fev. & $05 /$ mar. & 23 & 171 \\
\hline Grappolo 561 & $24 /$ set. & $17 /$ fev. & $28 /$ fev. & 11 & 157 \\
\hline Grappolo 575 & $05 /$ set. & $20 /$ fev. & $10 /$ mar. & 18 & 186 \\
\hline Negroa & $15 /$ set. & $10 /$ fev. & 03/mar. & 21 & 169 \\
\hline Penafiel & $10 /$ set. & 30/jan. & $23 /$ fev. & 24 & 166 \\
\hline Ropades 392 & $10 /$ set. & $10 /$ fev. & 03/mar. & 21 & 174 \\
\hline \multirow[t]{2}{*}{ Santa Catalina } & 20/set. & $20 /$ fev. & $15 /$ mar. & 23 & 176 \\
\hline & \multicolumn{5}{|c|}{ Ano agrícola 2009/2010 } \\
\hline Alto D’Ouro & 20/set. & $05 /$ fev. & $20 /$ fev. & 15 & 153 \\
\hline Arbequina & 30/ago. & $25 /$ jan. & $25 /$ fev. & 31 & 179 \\
\hline Galega & $03 /$ set. & $05 /$ fev. & $02 /$ mar. & 25 & 180 \\
\hline Grappolo 541 & $20 /$ set. & $10 /$ fev. & $28 /$ fev. & 18 & 161 \\
\hline Grappolo 561 & 25/ago. & $03 /$ fev. & $23 /$ fev. & 20 & 182 \\
\hline Grappolo 575 & 25/ago. & $20 /$ fev. & $05 /$ mar. & 13 & 192 \\
\hline Negroa & $25 /$ set. & $12 /$ fev. & $27 /$ fev. & 15 & 155 \\
\hline Penafiel & 30/ago. & $14 /$ fev. & $27 /$ fev. & 13 & 181 \\
\hline Ropades 392 & $05 /$ set. & $09 /$ fev. & $25 / \mathrm{fev}$ & 16 & 173 \\
\hline \multirow[t]{2}{*}{ Santa Catalina } & $10 /$ set. & $20 /$ fev. & $08 / \mathrm{mar}$. & 16 & 179 \\
\hline & \multicolumn{5}{|c|}{ Ano agrícola 2010/2011 } \\
\hline Alto D’Ouro & $30 /$ set. & $10 /$ fev. & $21 / \mathrm{fev}$ & 11 & 144 \\
\hline Arbequina & 25/ago. & $27 /$ jan. & $18 /$ fev. & 22 & 177 \\
\hline Galega & $17 /$ set. & $10 /$ fev. & 03/mar. & 21 & 167 \\
\hline Grappolo 541 & $15 /$ set. & $14 /$ fev. & $05 /$ mar. & 19 & 171 \\
\hline Grappolo 561 & $30 /$ set. & $10 /$ fev. & $23 /$ fev. & 13 & 146 \\
\hline Grappolo 575 & 25/ago. & $14 /$ fev. & $01 /$ mar. & 15 & 188 \\
\hline Negroa & 20/set. & $07 /$ fev. & $23 /$ fev. & 16 & 156 \\
\hline Penafiel & $20 /$ set. & $23 /$ fev. & $01 /$ mar. & 6 & 162 \\
\hline Ropades 392 & $20 /$ set. & $10 /$ fev. & $26 /$ fev. & 16 & 159 \\
\hline Santa Catalina & $10 /$ set. & $17 /$ fev. & $01 / \mathrm{mar}$. & 12 & 172 \\
\hline
\end{tabular}

IFR, início da frutificação; IC, início da colheita; FC, final da colheita; IC-FC, período de colheita; IFR-FC, período de frutificação. 
A escolha de cultivares adequadas às condições climáticas de cultivo local é fundamental, pois possibilita o escalonamento da produção e o aumento do período de oferta do produto ao mercado, o que favorece ainda a adaptação das tecnologias disponíveis à cultivar e região (Silva et al., 2006). Além disso, essa escolha proporciona diversas vantagens, tais como a redução no tempo de processamento após a colheita e a possibilidade de utilização dos equipamentos de extração por um período maior.

O diâmetro longitudinal dos frutos não apresentou diferença significativa entre as cultivares. Entretanto, houve diferença entre as cultivares quanto ao diâmetro transversal, que variou em até 56\% com 'Ropades 392' e 'Negroa', que apresentaram os menores valores, e 'Grappolo 561' que foi significativamente superior às demais (Tabela 3).

As cultivares Grappolo 541 e Santa Catalina apresentaram resultados significativamente superiores com relação à massa média total dos frutos; na porção polpa, além da 'Grappolo 541', destacaram-se também 'Grappolo 575' e 'Grappolo 561'. Quanto à massa das sementes, houve variação de até $125 \%$, e 'Santa Catalina' apresentou o maior valor, com $0,72 \mathrm{~g}$, e 'Negroa' o menor, com 0,32 g. No caso da escolha de cultivares de oliveiras destinadas à produção de frutos, tanto para a extração de azeite como para a conserva, deve-se dar preferência às cultivares que possuam caroços de tamanho reduzido e polpa com maiores

Tabela 3. Diâmetro longitudinal (DL) e transversal (DT) dos frutos, massa da polpa e sementes e teor lipídico da massa da polpa e caroço de cultivares de oliveira cultivadas em Maria da Fé, MG.

\begin{tabular}{|c|c|c|c|c|c|c|}
\hline \multirow[t]{2}{*}{ Cultivar } & \multirow{2}{*}{ DL } & \multirow{2}{*}{$\begin{array}{c}\text { DT } \\
\mathrm{mm} \text { )----- }\end{array}$} & \multicolumn{2}{|c|}{ Massa média (g) } & \multirow[t]{2}{*}{ Total } & \multirow{2}{*}{$\begin{array}{c}\text { Lipídeos } \\
(\%)\end{array}$} \\
\hline & & & Polpa & $\overline{\text { Semente }}$ & & \\
\hline AltoD’Ouro & $18,33 \mathrm{a}$ & $10,62 \mathrm{bcd}$ & $2,09 \mathrm{~cd}$ & $0,38 \mathrm{~cd}$ & $2,48 \mathrm{~cd}$ & $19,03 b$ \\
\hline Arbequina & $15,01 \mathrm{a}$ & $11,61 \mathrm{ab}$ & $0,90 \mathrm{f}$ & $0,57 \mathrm{~b}$ & $1,47 \mathrm{f}$ & $22,66 a$ \\
\hline Galega & $18,67 \mathrm{a}$ & $11,02 \mathrm{abc}$ & $1,91 \mathrm{~d}$ & $0,36 \mathrm{~cd}$ & $2,27 \mathrm{e}$ & $19,43 b$ \\
\hline Grappolo541 & $18,65 \mathrm{a}$ & $11,68 \mathrm{ab}$ & $2,69 \mathrm{a}$ & $0,46 b c$ & $3,14 \mathrm{a}$ & $16,34 \mathrm{~cd}$ \\
\hline Grappolo561 & $17,58 \mathrm{a}$ & $13,67 \mathrm{a}$ & $2,42 \mathrm{ab}$ & $0,40 \mathrm{~cd}$ & $2,83 \mathrm{~cd}$ & $22,35 \mathrm{a}$ \\
\hline Grappolo575 & $17,63 \mathrm{a}$ & $10,02 \mathrm{bcd}$ & $2,47 \mathrm{ab}$ & $0,37 \mathrm{~cd}$ & $2,85 \mathrm{bc}$ & $13,01 \mathrm{e}$ \\
\hline Negroa & $16,03 a$ & $8,31 \mathrm{~d}$ & $1,62 \mathrm{e}$ & $0,32 \mathrm{~d}$ & $1,94 \mathrm{~d}$ & $19,34 b$ \\
\hline Penafiel & $17,31 \mathrm{a}$ & $11,32 \mathrm{abc}$ & $2,07 d$ & $0,40 \mathrm{~cd}$ & $2,48 \mathrm{de}$ & $17,23 b c$ \\
\hline Ropades392 & $16,59 \mathrm{a}$ & $8,76 \mathrm{~cd}$ & $2,15 \mathrm{~cd}$ & $0,40 \mathrm{~cd}$ & $2,55 \mathrm{~d}$ & $14,32 \mathrm{de}$ \\
\hline Santa Catalina & $20,56 a$ & $12,06 \mathrm{ab}$ & $2,35 \mathrm{bc}$ & $0,72 \mathrm{a}$ & $3,08 \mathrm{ab}$ & $11,67 \mathrm{f}$ \\
\hline $\mathrm{CV}(\%)$ & 8,71 & 8,86 & 4,49 & 10,03 & 3,34 & 5,92 \\
\hline
\end{tabular}

${ }^{(1)}$ Médias seguidas de letras iguais, nas colunas, não diferem pelo teste de Tukey a $5 \%$ de probabilidade. dimensões, o que possibilitará maior rendimento no processamento (Oliveira et al., 2010a).

Em relação ao teor lipídico, houve variação de até 94,17\%, e 'Arbequina' e 'Grappolo 561' apresentaram resultados significativamente superiores às demais, com 22,7 e $22,4 \%$ de azeite, respectivamente (Tabela 3 ).

Apesar das menores dimensões dos frutos da cultivar Arbequina (Tabela 3), o rendimento da extração de azeite foi elevado, já que foram constatados $22,7 \%$ de lipídeos. Esses resultados discordam da afirmação, proposta por Oliveira et al. (2010a), de que as cultivares destinadas ao processamento devem possuir alta relação polpa/caroço. Por esses resultados, pode-se afirmar que, na escolha de cultivares promissoras para a extração de azeite, deve-se analisar o teor de lipídeos e não simplesmente as dimensões dos frutos.

Assim, as cultivares que apresentaram teores de lipídeos dentro do limite de 20 a 30\% foram Arbequina e Grappolo 561, as quais foram selecionadas para a etapa seguinte. Barranco et al. (2008) encontraram uma variação entre 17,1 e 22,5\% de lipídeos, em frutos das cultivares mantidas no Banco de Germoplasma de Córdoba, na Espanha, destinadas à extração de azeite.

Partindo-se da premissa de que a inclusão de duas cultivares em plantios comerciais aumenta o número de frutos por inflorescência (Suárez \& Rallo, 1987), as oliveiras 'Arbequina' e 'Grappolo 561' satisfariam este requisito, uma vez que há sincronia floral entre ambas (Tabela 1).

Apesar de a análise do perfil dos principais ácidos graxos não mostrar diferença significativa entre os tratamentos (cultivares e testemunha), altas percentagens de ácido oleico foram evidenciadas nas três amostras avaliadas: 'Arbequina', 61,3\%; 'Grappolo 561', 61,8\%; e espanhol, 62,4\%. Entre os ácidos graxos monoinsaturados, o principal é o ácido oleico, que possui propriedades que atuam na redução do colesterol total e LDL-c, sem reduzir o HDL-c. Além disso, causa alterações na membrana das plaquetas e produz ação antitrombótica (Vognild et al., 1998). Em relação ao ácido palmítico, palmitoleico e esteárico, os resultados apresentaram diferença significativa, e a cultivar Grappolo 561 foi superior nos três grupos avaliados, com 9,18, 3,45 e 0,70\% (Tabela 4). Quanto aoácidolinoleico, o azeite espanhol extravirgem apresentou-se significativamente superior aos demais, com $12,30 \%$ e, quanto ao linolênico, 'Arbequina' e o azeite espanhol comercial foram superiores. 
Tabela 4. Composição dos principais ácidos graxos - palmítico (PA), palmitoleico (PO), esteárico (ES), oleico (OL), linoleico (LO) e linolênico (LC) - presentes em cultivares de oliveiras ${ }^{(1)}$.

\begin{tabular}{|c|c|c|c|c|c|c|}
\hline \multirow[t]{2}{*}{ Cultivar } & PA & PO & ES & OL & LO & LC \\
\hline & \multicolumn{6}{|c|}{---------------------------(\%) } \\
\hline Arbe & $7,02 b$ & $2,43 b$ & $0,58 \mathrm{~b}$ & $61,30 \mathrm{a}$ & $10,58 b$ & $0,63 \mathrm{a}$ \\
\hline Grap & $9,18 \mathrm{a}$ & $3,45 \mathrm{a}$ & $0,70 \mathrm{a}$ & $61,78 \mathrm{a}$ & $11,22 \mathrm{ab}$ & $0,35 \mathrm{~b}$ \\
\hline spa & $05 b$ & $2,59 \mathrm{~b}$ & $0,58 b$ & $62,37 \mathrm{a}$ & $12,30 \mathrm{a}$ & $0,57 \mathrm{a}$ \\
\hline $\mathrm{V}(\%)$ & 5,56 & 11,77 & 15,40 & 1,49 & 3,75 & 7,80 \\
\hline
\end{tabular}

${ }^{(1)}$ Médias seguidas de letras iguais, nas colunas, não diferem pelo teste de Tukey, a 5\% de probabilidade.

\section{Conclusões}

1. O período de florescimento das diferentes cultivares varia de julho a outubro, e o de colheita de janeiro a março, em que a mais precoce é a Arbequina e as mais tardias são a Galega, a Grappolo 575 e a Santa Catalina.

2. 'Grappolo 561' apresenta frutos de maior diâmetro transversal, e 'Grappolo 541' frutos de maior massa total.

3. 'Arbequina' e 'Grappolo 561' apresentam maior teor de lipídeos.

\section{Referências}

BARRANCO, D. Variedades y patrones. In: BARRANCO, D.; FERNÁNDEZ-ESCOBAR, R.; RALLO, L. (Ed.). El cultivo del olivo. 6.ed. Madri: Mundi-Prensa-Junta de Andalucía, 2008. p.63-92.

BETTIOL NETO, J.E.; PIO, R.; SANCHES, J.; CHAGAS, E.A; CIA, P.; CHAGAS, P.C.; ANTONIALI, S. Produção e atributos de qualidade de cultivares de marmeleiro na região leste paulista. Revista Brasileira de Fruticultura, v.33, p.1035-1042, 2011.

CORDEIRO, A.M.; MORAIS, N.; ROGADO, B.; MATIAS, H.; SILVA, M.; RAMOS, A.E.; SIMÕES, P. Caracterização do período de floração de cultivares de oliveira (Olea europaea L.) em diferentes regiões. Resultados preliminares. Revista de Ciências Agrárias, v.24, p.32-37, 2001.

COUTINHO, E.F.; RIBEIRO, F.C.; CAPPELLARO, T.H. (Ed.). Cultivo de Oliveira (Olea europaea L.). Pelotas: Embrapa Clima Temperado, 2009. 125p. (Embrapa Clima Temperado. Sistemas de produção, 16).
HARTMAN, L.; LAGO, R.C. Rapid preparation of fatty acid methyl esters from lipids. Laboratory Pratice, v.22, p.475-476, 1973.

HORWITZ, W. (Ed). Official methods of analysis of AOAC International. $18^{\text {th }}$ ed. Maryland: AOAC International, 2005.

INDICADORES da agropecuária. Brasília: Companhia Nacional de Abastecimento, 2010. Disponível em: <http://www.conab.gov. br/conabweb/index.php?PAG=212>. Acesso em: 20 set. 2011.

OLIVEIRA, A.F. de; VIEIRA NETO, J.; GONÇALVES, E.D.; VILLA, F.; SILVA, L.F. de O. da. Parâmetros físico-químicos dos primeiros azeites de oliva brasileiros extraídos em Maria da Fé, Minas Gerais. Scientia Agraria, v.11, p.255-261, 2010a.

OLIVEIRA, M.C. de; VIEIRA NETO, J.; PIO, R.; OLIVEIRA, A.F. de; RAMOS, J.D. Enraizamento de estacas de oliveira submetidas à aplicação de fertilizantes orgânicos e AIB. Ciência e Agrotecnologia, v.34, p.337-344, 2010 b.

PETRI, J.L.; HAWERROTH, F.J.; LEITE, G. Fenologia de espécies silvestres de macieira como polinizadora das cultivares Gala e Fuji. Revista Brasileira de Fruticultura, v.30, p.868-874, 2008.

SANTOS, H.G. dos; JACOMINE, P.K.T.; ANJOS, L.H.C. dos; OLIVEIRA, V.A. de; OLIVEIRA, J.B. de; COELHO, M.R.; LUMBRERAS, J.F.; CUNHA, T.J.F. (Ed.). Sistema brasileiro de classificação de solos. 2.ed. Rio de Janeiro: Embrapa Solos, 2006. $306 \mathrm{p}$.

SILVA, R.P. da; DANTAS, G.G.; NAVES, R.V.; CUNHA, M.G. da. Comportamento fenológico de videira, cultivar Patrícia em diferentes épocas de poda de frutificação em Goiás. Bragantia, v.65, p.399-406, 2006.

SUÁREZ, M.P.; RALLO, L. Influencia de la polinización cruzada y del aclareo de inflorescencias y flores en la fructificación del olivo. ITEA, v.71, p.8-17, 1987.

TSIMIDOU, M. Polyphenols and quality of virgin olive oil in retrospect. Italian Journal of Food Science, v.10, p.99-116, 1998.

VIEIRA NETO, J.; OLIVEIRA, A.F. de; OLIVEIRA, N.C. de; DUARTE, H. da S.S.; GONÇALVES, E.D. Aspectos técnicos da cultura da oliveira. Belo Horizonte: Epamig, 2008. 56p. (Epamig. Boletim técnico, 88).

VIEIRA NETO, J.; SILVA, L.F. de O. da; LÚCIO, A.D.C.; OLIVEIRA, A.F. de; OLIVEIRA, M.C. Formulações comerciais de fertilizantes foliares na finalização de mudas de variedades de oliveira. Revista Ciência Agronômica, v.42, p.125-131, 2011.

VOGNILD, E.; ELVEVOLL, E.O.; BROX, J.; OLSEN, R.L.; BARSTAD, H.; AURSAND, M.; ØSTERUD, B. Effects of dietary marine oils and olive oil on fatty acid composition, platelet membrane fluidity, platelet responses, and serum lipids in healthy humans. Lipids, v.33, p.427-436, 1998.

Recebido em 20 de setembro de 2011 e aprovado em 8 de dezembro de 2011 